\title{
Condiciones laborales del personal de salud mental al comienzo de la pandemia por COVID-I 9 en Argentina
}

\author{
Working conditions among Mental Healthcare workers at the beginning of COVID-I9 \\ pandemic in Argentina
}

\section{Maximiliano Cesoni', Viviana A. Peskin², Diana Milena Berrio Cuartas ${ }^{3}$, Cora Luguercho $^{4}$}

\begin{abstract}
Resumen
Introducción: El contexto de pandemia por COVID-19 subraya y resalta las distintas dificultades de los trabajadores de la salud. La Asociación de Psiquiatras Argentinos (APSA) confeccionó una encuesta, que se difundió entre trabajadores de la Salud Mental. El objetivo del estudio fue medir y conocer cuáles eran las condiciones laborales del personal que se desempeñó en el campo de la Salud Mental en Argentina al comienzo de la pandemia. Método: Se realizó un estudio descriptivo transversal en una muestra de conveniencia. A tal fin se diseñó una encuesta, en formato digital,y se difundió desde APSA. Esta constaba de 27 preguntas y una opción de comentarios, incluyendo variables sociodemográficas, datos específicos de la labor desempeñada y actos de discriminación padecidos. Resultados: La encuesta fue respondida por 710 personas. $32 \%$ de las respuestas provenientes de trabajadores del sector público, indicaron que debían proveer su propio equipo de protección personal, comparado con $56 \%$ en el sector privado. Cerca del $82 \%$ de las respuestas del sector público indicaron que existían protocolos para pacientes con síntomas compatibles con COVID-19, en comparación con $58 \%$ en el sector privado. Para pacientes nuevos en una internación, las respuestas desde el sector público indicaron que más del $50 \%$ tenía protocolos de manejo, en tanto menos de la mitad en el privado. Conclusiones: Los resultados de la encuesta permiten conocer cuáles fueron las condiciones laborales al comienzo de la pandemia por COVID-19 en la Argentina. Tener un plan en función de lo que se sabe y se tiene, permite un abordaje más adecuado, para los trabajadores y para quienes dependen de ellos en los cuidados.
\end{abstract}

Palabras clave: Salud Mental - Condiciones laborales -Pandemia COVID-19 - Profesionales de Salud Mental y pandemia.

\begin{abstract}
Introduction: The current COVID-19 pandemic highlights the different difficulties that healthcare workers have to face in this context. In order to quantify some aspects of the current working situation, the Asociación de Psiquiatras Argentinos (APSA) designed a survey for Mental Healthcare workers. The goal was to: measure and know what are the working conditions of those working in Mental Health in Argentina, in this pandemic context. Methods: Cross sectional descriptive study. Convenience sample. The survey was designed and sent in a digital format, thru APSA means of communication. The survey had 27 questions and one comments option. Questions explored sociodemographic variables, worked related aspects and discrimination suffered by participants. Results: The survey, in its digital format, was answe-

\footnotetext{
RECIBIDO |3/6/202| - ACEPTADO |4/9/202|

1. Médico de planta del Hospital “José T. Borda”. Prosecretario de la Asociación de Psiquiatras Argentinos (APSA).

2. Médica Especialista en Psiquiatría (UBA). Coordinadora Revista Sinopsis Digital. Miembro del Capítulo Investigación en Psiquiatría (APSA).

3. Médico Psiquiatra (APSA). Psiquiatra de consulta externa Hospital Británico de Buenos Aires. Miembro del Capítulo de Psiquiatría antropológica de APSA, Coordinadora Revista Sinopsis Digital.

4. Secretaria de la Asociación de Psiquiatras Argentinos (APSA). Especialista en psiquiatría. Ex directora de Salud Mental comunitaria de Chubut. Presidenta honoraria de la Asociación de Psiquiatras del Chubut (APCHU).
} 
red by 710 participants. $32 \%$ of those in the public sector pointed out that people needed to bring their own personal protective equipment, compared to $56 \%$ in the private sector. For protocols in place, nearly $82 \%$ of public sector responses said there is a protocol for COVID-19 compatible symptoms in patients, compared to $58 \%$ in the private sector. And for new patients in an inpatient facility, responses showed that more than $50 \%$ had a protocol in the public sector, vs less than half in the private setting. Conclusions: This survey and the results allows to know and have evidence on which were the working conditions at the beginning of the pandemic. Having a plan considering what is known and what is available, allows a more appropriate approach, for both the workers and those who depend on them for care.

Keywords: Mental Health - Working conditions - Pandemic COVID-19 - Mental Health workers and pandemic.

\section{Introducción}

El contexto actual de la pandemia por COVID-19 pone de relieve, y acentúa, distintas realidades sobre las condiciones laborales de los trabajadores de la salud en la Argentina. Más allá de la percepción subjetiva de los distintos profesionales, que surge en los intercambios informales entre colegas, el poder objetivarlas permite conocer esa realidad, y pensar mejores estrategias para abordarla.

El ámbito de desempeño de los profesionales de la salud es muy importante tanto para los mismos, como para la población que recibe su atención. En los últimos años se ha investigado mucho acerca del tema y se ha observado que un ambiente laboral adverso, genera efectos negativos físicos y mentales a largo plazo (Kapinos et al, 2012; Sobrequés et al, 2003; West et al, 2018; WPA, 2018).

Una de las entidades clínicas descripta es el síndrome de burnout, con estudios realizados tanto a nivel mundial y local en distintas profesiones vinculadas a las tareas asistenciales en salud (Berrio Cuartas et al, 2017; Bianchini Matamoros, 1997; Bonadeo et al, 2005; Kumar, 2007; Peckham, 2015; Wolfberg, 2003). En 2017, el Capítulo de Psiquiatras en Formación de la Asociación de Psiquiatras Argentinos (APSA), publicó una investigación acerca de la prevalencia del síndrome de burnout entre los psiquiatras, y otra, publicada en 2018, sobre distintas características vinculadas a las condiciones laborales de los mismos (Berrio Cuartas et al, 2017; Cesoni et al, 2018).

La irrupción inesperada del virus SARS-COV 2 ha tenido un impacto en la salud mental y general de la población (Ardila Gómez et al, 2021; Johnson et al, 2020; Sánchez Duque et al, 2020). La Organización Mundial de la Salud declaró que el mundo se encontraba atravesando una pandemia en marzo de 2020 (OPS, 2020). Los servicios de atención en salud de todo el mundo no estaban capacitados para responder frente a la amplia demanda de recursos humanos y técnicos, sumados a la falta de suministros. Además, dada la poca información acerca de la nueva enfermedad, las medidas de contención eran inciertas, lo cual generaba incertidumbre tanto en la población, como entre los profesionales a cargo de su cuidado y de la toma de decisiones. En ese contexto sanitario, se encontró una gran dificultad para planificar, organizar estrategias de protección para evitar contagios y proveer los elementos necesarios para que el personal de salud pudiera realizar su tarea sin aumentar su propio riesgo de contraer la enfermedad (Ardila Gómez et al, 2021).

Los trabajadores del campo de la Salud Mental se vieron enfrentados a muchos cambios en sus prácticas laborales. Algunos profesionales, dadas las características de la disciplina, pudieron continuar la atención a distancia, utilizando distintos recursos digitales. Sin embargo, no siempre esa opción fue posible porque su utilización dependía de múltiples variables que la dificultaban (Stewart \& Appelbaum, 2020).

A partir de los relatos de las experiencias y preocupaciones de los profesionales del campo de la Salud Mental, desde APSA se realizó una encuesta cuyo objetivo fue conocer cuáles eran las condiciones laborales del personal que se desempeñaba en ese área en la Argentina en tan difícil contexto, a fin de proponer estrategias, para mejorar su labor y brindarles herramientas adecuadas.

\section{Materiales y métodos}

Se realizó una investigación de corte transversal y descriptiva. A tal efecto, un equipo de investigación de APSA diseñó una encuesta que fue administrada en forma anónima y voluntaria, distribuida en formato digital por el boletín de APSA y sus redes sociales entre los meses de abril y junio de 2020. Muestra de conveniencia.

La encuesta constaba de 27 preguntas y una opción de comentarios al final (ver Anexo 1). 
Las primeras preguntas exploraban características sociodemográficas (edad, sexo, profesión, nacionalidad, provincia, ciudad y sector donde ejercían los encuestados). También contenía preguntas específicas de la labor desempeñada en el contexto de la pandemia (atención presencial o a distancia, cumplimiento de las excepciones para las tareas asistenciales presenciales por parte del empleador y pago del salario, medidas de desinfección en el ámbito laboral y sector de desempeño, equipo de protección personal, quién lo proveía, protocolos de manejo para pacientes con sintomatología de COVID-19 en la admisión, cumplimiento del mismo, existencia y cumplimiento de protocolos por COVID-19 en salas de internación). Finalmente se consultó sobre si el encuestado había padecido algún acto de discriminación por su labor como personal de salud.

La encuesta fue respondida por 710 personas que se desempeñaban laboralmente en el campo de la Salud Mental, entre ellos: psiquiatras, psicólogos/as, trabajadores sociales, acompañantes terapéuticos/as, administrativo/a, enfermeros/as, farmacéuticos/as, psicopedagogos/as, terapistas ocupacionales, musicoterapeutas, nutricionista, médicos/as de otras especialidades, sociólogos/as, psicólogo/a social.

\section{Resultados}

La edad promedio de aquellos que respondieron la encuesta fue de 44,3 años (edad mínima: 24 años, edad máxima: 80 años). 517 de los encuestados se identificaron de género femenino (72,8\%), 191 de género masculino (26,9\%) y 2 no especificaron el género. La muestra incluyó: 474 psiquiatras $(66,7 \%), 168$ psicólogos/as (23,7\%), 16 trabajadores/as sociales (2,3\%), 1 administrativo/a, 5 acompañantes terapéuticos/as, 2 farmacéuticos/as, 18 enfermeros/as, 4 terapistas ocupacionales, 2 musicoterapeutas, 1 nutricionista, 1 psicólogo/a social, 3 psicopedagogos/as, 1 psicoterapeuta, 11 médicos/as de distintas especialidades, 1 sociólogo/a.

En la tabla 1 se detalla la distribución por provincia de los profesionales que respondieron la encuesta.

En relación al sector donde se desempeñaban los participantes de la encuesta, 275 (38,7\%) refirieron realizar sus tareas tanto en el sector público como en el privado, 220 (31\%) sólo en el privado, 213 (30\%) sólo en el público y 2 en otros.

Dentro del sector público, 402 (82,3\%) contestaron que realizaban su trabajo en forma presencial. En el caso del sector privado, 181 (43\%) respondieron también que asistían a su ámbito laboral.
Tabla I.

\begin{tabular}{lc}
\hline Regiones y Provincias & $\begin{array}{c}\text { Cantidad de } \\
\text { respuestas }\end{array}$ \\
\hline Buenos Aires (CABA, Provincia de Buenos Aires) & 437 \\
\hline Centro (Córdoba, La Pampa) & 25 \\
\hline Cuyo (Mendoza, San Juan, San Luis) & 81 \\
\hline Litoral (Santa Fe, Entre Ríos) & 41 \\
\hline Noreste (Misiones, Corrientes, Chaco, Formosa) & 71 \\
\hline $\begin{array}{l}\text { Noroeste (Jujuy, Salta, Catamarca, La Rioja, } \\
\text { Tucumán, Santiago del Estero) }\end{array}$ & 28 \\
\hline $\begin{array}{l}\text { Patagonia (Chubut, Río Negro, Neuquén, Santa } \\
\text { Cruz,Tierra del Fuego) }\end{array}$ & 27 \\
\hline
\end{tabular}

Frente a la consulta sobre el origen del equipo de protección personal, 466 encuestados pertenecientes al sector público respondieron de la siguiente manera: 231 (50\%) indicaron que fueron provistos en el lugar de trabajo, 150 (32\%), tuvieron que proveerse a su costo y solamente 52 (cerca del 11\%) los obtuvieron por medio de donaciones, el resto de las respuestas indican múltiples orígenes.

Sobre el origen del equipo de protección personal en el sector privado respondieron 337 profesionales; 132 (cercano al 39\%) indicaron que los proveía el empleador, 189 (56\%) contestaron que debía procurárselos el trabajador, $6(1,8 \%)$ lo obtuvieron por donaciones, y $10(3,2 \%)$ los obtuvo de múltiples orígenes.

Ante la pregunta de si existía un protocolo de manejo para pacientes que se presentaban con alguno de los síntomas descritos de COVID-19, 391 (82\%) profesionales del sector público y 200 (58\%) del sector privado, indicaron que sí.

Frente al ingreso de nuevos pacientes en una institución de internación, desde el sector público han respondido que hay un protocolo 229 (57\%) participantes, pero desde el sector privado 98 (39\%) han indicado que existe una guía de aislamiento para pacientes nuevos.

Cuando se preguntó a los participantes de la encuesta si les resultaban suficientes las medidas de desinfección tomadas en su ámbito laboral, 164 (35\%) del sector público y 196 (53\%) del sector privado respondieron que sí.

En la tabla 2 se encuentran las respuestas en relación a haber padecido actos de discriminación, agresiones verbales o físicas a raíz de su condición de trabajador de la salud.

Al final de la encuesta, fue incluida una opción de comentarios. 
Tabla 2.

\begin{tabular}{lccc}
\hline Preguntas & Sí & No & $\begin{array}{c}\text { No sabel } \\
\text { No contesta }\end{array}$ \\
\hline $\begin{array}{l}\text { iHa sufrido algún acto } \\
\text { de discriminación, por su } \\
\text { condición de profesional de } \\
\text { la salud, en la vía pública? }\end{array}$ & 5 I & 628 & 8 \\
\hline $\begin{array}{l}\text { ¿Ha sufrido algún acto } \\
\text { de discriminación en su } \\
\text { vivienda? }\end{array}$ & 33 & 647 & 7 \\
\hline $\begin{array}{l}\text { iHa sufrido alguna agresión } \\
\text { verbal por su condición } \\
\text { de profesional de la salud } \\
\text { desde el inicio de la pan- } \\
\text { demia? }\end{array}$ & 57 & 628 & 3 \\
\hline $\begin{array}{l}\text { iHa sufrido alguna agre- } \\
\text { sión física por su condi- } \\
\text { ción de profesional de la } \\
\text { salud desde el inicio de la } \\
\text { pandemia? }\end{array}$ & 7 & 677 & \\
\hline
\end{tabular}

\section{Discusión}

A partir de la preocupación reinante en el seno de APSA por las noticias sobre las condiciones de trabajo de los colegas se decide realizar una encuesta intentando reflejar las condiciones laborales del personal que se desempeñaba en el campo de la Salud Mental en Argentina en 2020. La inquietud ante la llegada de la pandemia, se dio en el marco de un panorama laboral ya problemático que se había dado a conocer en el año 2018 a partir de una investigación realizada por el Capítulo de Psiquiatras en Formación de APSA (Cesoni et al, 2018).

Este estudio presenta limitaciones, vinculadas al modo de selección de la muestra, que podría introducir un sesgo en los resultados observados. Aquellos/as con mayores deseos de visibilizar el malestar en su ámbito laboral, podrían llevar a una sobrevaloración de ciertas dificultades planteadas. Sin embargo, dado el $\mathrm{n}$ de participación y la característica anónima de la encuesta, entendemos que los resultados aquí observados son relevantes, y sobre los cuales hay que pensar en estrategias a futuro. Se podrían pensar también, análisis estadísticos posteriores sobre los resultados obtenidos, de orden inferencial, centrados en explorar las respuestas por profesión, sector de desempeño, lugar de ejercicio, entre otros... Creemos que esta presentación, meramente descriptiva de los datos, permitirá, a partir de los intercambios que en torno a ellos se genere, futuros estudios y reevaluación de los datos aquí presentados.

En septiembre de 2020, a posteriori de la realización de la encuesta, se publicó una declaración de la directora de la Organización Panamericana de la Salud (OPS), Carissa F. Etienne, que afirmaba: "El personal de la salud es especialmente vulnerable a la pandemia del COVID-19, y en la Región de las Américas, tenemos la mayor cantidad de trabajadores de la salud infectados en el mundo" (Naciones Unidas, 2020). Se suma al riesgo de contagio, las consecuencias de desarrollar las tareas entre tanta incertidumbre, con guías en construcción, que se iban y van modificando en función de la nueva evidencia disponible, pero que daba (y da) cuenta de todo lo desconocido. No había evidencia suficiente para construir conocimiento sobre el cual apoyarse para la toma de decisiones. Un claro ejemplo de esto fueron las discusiones en torno al uso o no de tapabocas. Era necesario, no lo era, hubiera sido deseable pero escaseaba, cómo decidir quién tenía que usarlo y quién no. Vacunación mediante, continúa el debate en este sentido.

Entre los profesionales de la salud, surgieron preocupaciones sobre la falta de entrenamiento en el uso del equipo de protección personal, ya que muchos desempeñaban sus tareas en consultorios externos $\mathrm{u}$ otros ámbitos laborales diferentes a los que cumplían antes de la pandemia. En este sentido, algunos profesionales fueron enviados a realizar actividades no vinculadas con su formación como psiquiatras. De los encuestados en esta oportunidad, la mayoría de los que se desempeñaban en el ámbito público, asistían presencialmente a sus lugares de trabajo (82,3\% vs $43 \%$ en el ámbito privado). Estos resultados nos permiten considerar que el personal que se desempeña en el sector público estuvo y está más expuesto al riesgo de contagio. Se requerirían estudios más específicos respecto a las condiciones laborales por sector y cómo se modificaron a lo largo de la pandemia.

Se observa cierta variabilidad en torno a la presencia de protocolos para el manejo de personas que consultan en Salud Mental con síntomas compatibles con COVID-19, al igual que en el caso de las internaciones. El sector público presenta más respuestas dando cuenta de la existencia de protocolos, en comparación con el sector privado. Las guías de manejo son herramientas muy importantes en estos contextos, donde la comunicación clara y concisa, permite una mejor toma de decisión por parte de los profesionales de la salud actuantes. Sería interesante ver, ya transcurrido más de un año de la encuesta, si estos protocolos se encuentran presentes, y explorar si son conocidos por los trabajadores de la salud de cada institución. La comunicación intrainstitucional es fundamental para una respuesta ordenada frente a la incertidumbre. 
Durante la pandemia, en todo el mundo, las prestaciones en salud, que por su motivo de consulta lo permitiera, han podido realizarse a distancia (de manera virtual) y han estado indicadas como medida preferencial desde que se instaló el Aislamiento Social Preventivo Obligatorio (Boletín oficial de la República Argentina), resguardando al paciente, pero también al profesional. Como fue mencionado, eso dependía y depende de las características de la patología a tratar, $\mathrm{y}$ de ciertos aspectos socio-culturales de quien consulta (el acceso a internet y tecnológico a la atención, contar con espacio de intimidad suficiente para recibir la atención específica, entre otras) (Markowitz et al., 2020). De más está decir que la atención en forma remota no escapa a la brecha digital (Negueruela \& Torres, 2020) que se ve reflejada a nivel social en el acceso a la escolarización, al trabajo remoto, etc.

Es de importancia mencionar que algunas investigaciones dan cuenta de los efectos en la Salud Mental de la población general en el contexto de pandemia: ansiedad, depresión, estrés postraumático, insomnio, irritabilidad y estados conductuales alterados, incluso violencia (Johnson et al., 2020; Ardila Gómez et al., 2021; Taquet et al., 2020; Stewart y Appelbaum, 2020). Dentro de las principales cuestiones que mencionan, sugieren que los psiquiatras continúen con los tratamientos individuales y grupales de sus pacientes e incluso intenten el cuidado de sus colegas en cuanto al mantenimiento de hábitos y rutinas saludables, horas de sueño, ejercicio, entre otras y consultar a un profesional de la salud mental si comienzan a tener ansiedad, depresión, u otros síntomas de la esfera psíquica (Stewart \& Appelbaum, 2020). Los trabajadores de la salud se encuentran, al igual que la población general, en riesgo de desarrollar la sintomatología mencionada. En este punto, varias asociaciones e instituciones del campo de la Salud Mental, brindaron su saber, su experiencia, para acompañar a aquellos colegas que se encontraban a diario sosteniendo a los pacientes con COVID-19 y sus familiares. Esos puentes trazados, tanto en Argentina como en otros lugares del mundo, permitieron una construcción solidaria para pensar en una respuesta desde el entretejido social, cada uno sumando desde sus saberes y recursos. Se vieron ejemplos de estas iniciativas en distintos ámbitos como fábricas que transformaban su producción a barbijos, otras a mamparas, a guantes, mecanismos de sanitización, etc...

Así como mencionamos, varias situaciones de construcción social colectiva para hacer frente a la pandemia, nos pareció importante conocer si los profesionales habían sufrido situaciones de discrimina- ción o maltrato por su ámbito laboral. Consideramos que merece ser más estudiado en el futuro y ver cómo fue evolucionando. Son variadas las situaciones presentadas, las cuales sucedieron en muchos lugares del mundo. La población, al tener gran temor al contagio, rechazó, en algunos contextos, el contacto con personal médico, aquellos que realizaban tareas asistenciales o en ámbitos de atención en pandemia (INADI, 2020; Valdés et al., 2020).

Entre los encuestados, 51 participantes reportaron vivir actos de discriminación en la vía pública por ser profesionales de la salud, 33 actos de discriminación en su vivienda y 57 han contestado haber sufrido alguna agresión verbal por su condición de profesional de la salud desde el inicio de la pandemia. Inclusive, 7 participantes de los encuestados, han reportado haber sufrido alguna agresión física desde el inicio de la pandemia.

Nos parece importante dar cuenta del total de participantes que reportaron estos hechos. Así como el miedo puede promover el armado de lazos, pensar en estrategias colectivas para una salida del contexto de crisis, también el miedo puede llevar a buscar en el otro, en el par, la amenaza. Surge la pregunta, desde el campo de la Salud Mental, de cómo trabajar para pasar de un "otro amenazante", a un "otro par" con quien pensar e implementar una salida conjunta.

\section{Conclusiones}

La decisión de realizar la presente encuesta, con el objetivo de conocer y cuantificar algunos aspectos de las condiciones laborales de los profesionales de la Salud Mental fue el primer paso para hacer un diagnóstico, y construir soluciones en un momento de incertidumbre. A pesar de haber sido realizada al comienzo de la pandemia, en donde el contexto y la realidad era otra, el gran dinamismo de la situación actual enseña que, de lo sucedido en Argentina y en otros países, se aprende a pensar estrategias a futuro. Pasar del lugar de queja, a la exploración, conocimiento, y construcción, permite pensar mejores estrategias en donde anclarse. Cada iniciativa en pos del diseño de protocolos, guías, comunicación, construye, organiza, arma lazos, todos ellos fundamentales para atravesar y acompañarnos en este territorio inexplorado. En este sentido, consideramos que nuevos estudios sobre las condiciones laborales de los profesionales en el ámbito de la Salud Mental en Argentina, permitirán reevaluar las variables y objetivar los funcionamientos de las estrategias planteadas.

Conocer las condiciones laborales que se presentaban en ese primer momento de la pandemia habi- 
litan a reflexionar, revisar y aprender. Los resultados nos permiten tener un parámetro objetivo, tanto para el personal que desempeña sus tareas laborales en la Salud Mental, como para quienes son responsables de garantizar las condiciones adecuadas de las mismas.

\section{Conflictos de intereses: los autores declaran no tener conflictos de intereses.}

Agradecimientos: Agradecemos a la Asociación de Psiquiatras Argentinos, y en especial a su presidente, Santiago Levin, por permitirnos y alentarnos a llevar adelante esta investigación.

\section{Referencias bibliográficas}

Ardila Gómez, S., Rosales, M. L., Fernández, M. A., Velzi Díazm A., Matkovich, A., Agrest, M. (2021). Impacto de la pandemia por COVID-19 en los servicios de salud mental en Argentina. Rev Argent Salud Publica, 13(Supl. COVID-19:e26). http://rasp.msal.gov.ar/rasp/articulos/vol13supl/AO Ardilae26.pdf

Berrio Cuartas, D. M., Mazzoglio y Nabar, M., Peskin, V., Furman, M., Falicoff, J., Muñiz, M., Reynoso, F., Diez, C. (2017). Burnout en psiquiatras en formación a nivel nacional. Revista Intra Med, 6(3). https://www.intramed. net $/$ contenidover.asp? contenidoid $=91835$ \&pagina $=1$

Bianchini Matamoros, M. (1997). El Síndrome del Burnout en personal profesional de la salud. Medicina Legal de Costa Rica, 13-14(2-12),189-192.http://www.scielo.sa.cr/scielo.php?script=sci arttext\&pi$\mathrm{d}=$ S1409-00151997000200017\&lng=en\&tlng=es.

Boletín oficial de la República Argentina. Aislamiento Social Preventivo y Obligatorio (ASPO),3 de julio, 2021. https://www.boletinoficial.gob.ar/ detalleAviso/primera/227042/20200320\%20agregar\%20

Bonadeo, M. A., Marenghi, M., Bassi, F., Fernández, M. E., Lago, M. I., Piacentini, A., Rodríguez Bertoni, C. (2005). Evaluación del grado de satisfacción del personal médico en un hospital de pediatría. Arch argent pediatr, 103(6), 491496. https://www.sap.org.ar/docs/archivos/2005/arch05_6/491.pdf

Cesoni, M., Peskin, V. A., BerrioCuartas, D. M., Tenconi, J. C., Furman, M., Pereyra, W., Furman, M., Ibañez, B., Baratti, M., Lagos, D., García, N., Mendieta, M., Sanchez, F. (2018). Condiciones Laborales de los psiquiatras en Argentina. Vertex Rev Arg Psiquiatr, 29(140), 299- 306.

Instituto Nacional contra la Discriminación (INADI). (2020). La xenofobia y el racismo. Discriminación y Coronavirus. https://www.argentina. gob.ar/sites/default/files/discriminacion_y_coronavirus.pdf

Johnson, M. C., Saletti-Cuesta, L., Tumas, N. (2020). Emociones, preocupaciones y reflexiones frente a la pandemia del COVID-19 en Argentina. Ciência \& Saúde Coletiva, 25(Supl.1), 2447-2456. doi.org/10.1590/1413$\underline{81232020256.1 .10472020}$

Kapinos, K. A., Fitzgerald, P., Greer, N., Rutks, I. (2012). The Effect of Working Conditions on Patient Care: A Systematic Review [Internet]. Washington (DC): Department of Veterans Affairs (US). https://www.ncbi.nlm. nih.gov/books/NBK114447
Kumar, S. (2007). Burnout in psychiatrists. World Psychiatry, 6(3), 186-189. https://www.ncbi.nlm.nih.gov/pmc/articles/PMC2175073/?report=classic

Markowitz, J.C., Milrod, B., Heckman, T. G., Bergman, M., Amsalem, D., Zalman, H., Ballas, T., Neria, Y. (2021). Psychotherapy at a Distance. Am J Psychiatry, 178:3. https://doi.org/10.1176/appi.ajp.2020.20050557

Naciones Unidas. Información Oficial México (2020, 2 de septiembre). Cerca de 570.000 trabajadores de la salud se han infectado y 2.500 han muerto por COVID-19 en las Américas. https://coronavirus.onu.org.mx/ cerca-de-570-000-trabajadores-de-la-salud-se-han-infectado-y-2-500han-muerto-por-covid-19-en-las-americas

Negueruela, A., Torres, B. (2020). La brecha digital impacta en la educación. https://www.unicef.es/educa/blog/covid-19-brecha-educativa

Organización Panamericana de la Salud. (2020, 11 de marzo). La OMS caracteriza a COVID-19 como una pandemia. https://www.paho.org/es/ noticias/11-3-2020-oms-caracteriza-covid-19-como-pandemia

Peckham, C. (2015). Medscape Psychiatrist lifestyle report. http://www. medscape.com/features/slideshow/lifestyle/2015/psychiatry?src=wnl edit_specol\&uac $=198826 \mathrm{ET} \# 1$

Sánchez Duque, J. A., Arce Villalobos, L. R., Rodríguez Morales, A. J. (2020). Enfermedad por coronavirus 2019 (COVID-19) en América Latina: papel de la atención primaria en la preparación y respuesta. Aten Primaria, 52(6), 369-372. https://doi.org/10.1016/j.aprim.2020.04.001

Sobrequés, J., Cebriàa, J., Segurac, J., Rodríguez, C., García, M., Juncosa, S.(2003). La satisfacción laboral y el desgaste profesional de los médicos de atención primaria. Aten Primaria, 31(4), 227-33. https://doi.org/10.1016/ $\underline{\mathrm{S} 0212-6567(03) 791}$

Stewart, D. E., Appelbaum, P. S. (2020). COVID-19 and psychiatrists' responsibilities: a WPA position paper. World Psychiatry, 19(3), 406-407. https://doi.org/10.1002/wps.20803

Taquet, M., Sierra, L., Geddes, J. R., Harrison, P. J. (2020). Bidirectional associations between COVID-19 and psychiatric disorder: retrospective cohort studies of 62354 COVID-19 cases in the USA. www.thelancet.com/ psychiatryhttps://doi.org/10.1016/S2215-0366(20)30462-4

Valdés, P. R., Cámera, L. A., De la Serna, M., Abuabara Turbay, Y., Carballo Zárate, V., Hernández Ayazo, H., Sierra Merlano, R. M., Viera Jaraba, A., Rodríguez Hurtado, D., Vaucher Rivero, A., Melgar Cuéllar, F., Ibáñez Guzmán, C., Araya Fonseca, C., Betancourt-Torres, I., Montúfar Guardado, R., Nitsch Montiel, C., Brav Mejía, C. R., Salgado Guevara, D. A., Bustillo y Valeriano, P. L.(...), Carrasco Dueñas, S. Foro Internacional de Medicina Interna (FIMI). (2020). Ataque al personal de la salud durante la pandemia de COVID-19 en Latinoamérica. Acta Med Colomb, 45(3), 1-10. doi:https://doi.org/10.36104/amc.2020.1975

West, C. P., Dyrbye, L. N., Shanafelt, T. D. (2018). Physician burnout: contributors, consequences and solutions. Journal of Internal Medicine, 283(6), 516-529. https://doi.org/10.1111/joim.12752

Wolfberg, E. (2003). Crisis social y desgaste ocupacional de los profesionales de la salud: alertas y recursos. Vertex Rev Arg Psiquiatr, 14(54), 268-279.

World Psychiatric Association (WPA). (2008). Entornos positivos para la práctica de los profesionales de atención de salud. http://www.whpa.org/ PPE_General information_Sp.pdf 


\section{ANEXO I}

Encuesta sobre condiciones laborales durante la Pandemia por COVID-I 9 en profesionales de Salud Mental en Argentina Esta encuesta es realizada por la Asociación de Psiquiatras Argentinos (APSA) con el objetivo de conocer las condiciones en las cuales los profesionales de la Salud Mental en Argentina se encuentran trabajando durante la Pandemia por COVID-19. Se incluyen preguntas demográficas y geográficas sobre el lugar de trabajo y sobre si garantizan las condiciones y elementos de protección personal (EPP) tanto en INSTITUCIONES públicas como privadas.

Es una encuesta ANÓNIMA y VOLUNTARIA.AI completarla autoriza el uso de la información con fines investigativos.

Si tiene consultas sobre esta encuesta contactarse a apsavirtual@gmail.com

I. Edad (en años):

2. Sexo: Masculino $\square \quad$ Femenino $\square \quad$ Otro $\square$

\section{Profesión:}

Psiquiatra $\square \quad$ Residente de psiquiatría $\square \quad$ Concurrente de psiquiatria $\square \quad$ Psicólogo /a $\square$ Residente de psicología $\square \quad$ Concurrente de psicología $\square \quad$ Enfermero $\square \quad$ Trabajador social $\square$ Residente de trabajo social $\square$

Otra:

\section{Nacionalidad :}

5. ¿En qué provincia de Argentina ejerce principalmente?

\begin{tabular}{|c|c|c|c|c|c|c|}
\hline \multirow{2}{*}{\multicolumn{2}{|c|}{$\begin{array}{l}\text { Provincia de Buenos Aires } \square \\
\text { Córdoba } \square \quad \text { Corrientes }\end{array}$}} & \multicolumn{2}{|c|}{ Ciudad Autónoma de Buenos Aires $\square$} & \multirow{2}{*}{$\begin{array}{l}\text { Catamarca } \square \\
\text { La Pampa } \square\end{array}$} & \multirow{2}{*}{$\begin{array}{l}\text { Chaco } \square \\
\text { La Rioja } \square\end{array}$} & \multirow{2}{*}{$\begin{array}{l}\text { Chubut } \square \\
\text { Mendoza } \square\end{array}$} \\
\hline & & Entre Ríos $\square$ & Formosa $\square$ & & & \\
\hline$\square$ & Neuquén $\square$ & Rio Negro $\square$ & Salta $\square$ & $\square$ & & uz $\square$ \\
\hline
\end{tabular}
Misiones $\square \quad$ Neuquén $\square \quad$ Rio Negro $\square \quad$ Salta $\square \quad$ San Juan $\square \quad$ San Luis $\square \quad$ Santa Cruz $\square$ Santa Fe $\square \quad$ Santiago del Estero $\square \quad$ Tierra del Fuego $\square \quad$ Tucumán $\square$

6. ¿En qué ciudad ejerce principalmente?

7. ¿En qué sector(es) ejerce? (puede marcar más de uno) Público $\square \quad$ Privado $\square \quad$ Otro $\square$

TRABAJO EN EL SECTOR PÚBLICO (en caso de no trabajar en el sector público avance a la pregunta I6)

8. ¿Está asistiendo a su lugar de trabajo de forma presencial?

Sí $\square \quad$ No $\square$

8. I. ¿Su empleador cumple con exceptuar de realizar tareas clínico/asistenciales a profesionales que se encuentren dentro de la población de riesgo para COVID-19 o a cargo de un menor? (Población de riesgo: embarazadas, comorbilidades).

Sí $\square \quad$ No $\square \quad$ No sabe/ no contesta $\square$

8.2.En caso de estar exceptuado de asistir a su lugar de trabajo ¿su empleador continúa abonando su sueldo completo?

Sí $\square \quad$ No $\square \quad$ No sabe/ no contesta $\square$

9. ¿Le resultan suficientes las medidas de desinfección tomadas en su ámbito laboral? (limpieza de consultorios con productos adecuados, limpieza de áreas de uso común entre profesionales, etc.)

Sí $\square \quad$ No $\square \quad$ No sabe / no contesta $\square$

10. Su ámbito de trabajo es:

Institución monovalente en Salud Mental $\square \quad$ Institución polivalente $\square \quad$ Otra $\square$

I I. ¿En qué sector del ámbito se desempeña? (puede seleccionar más de una opción)

Guardia $\square \quad$ Servicio de internación $\square \quad$ Comunidad Terapéutica $\square \quad$ Consultorios Externos $\square \quad$ Hospital de día $\square$ Centro de salud $\square \quad$ Hogar de adultos mayores $\square \quad$ Otra $\square$

I 2. ¿Cuál de los siguientes elementos tanto del equipo de protección personal (EPP) como de higiene cuenta en su trabajo? Barbijo $\square \quad$ Camisolín $\square \quad$ Guantes $\square \quad$ Protección ocular $\square$ Máscaras (protección plástica transparente que cubre la cara) $\square \quad$ Tapa nariz y boca $\square$

Acceso a agua potable y jabón entre pacientes $\square \quad$ Alcohol en gel $\square \quad$ Ninguno $\square \quad$ Otro $\square$

13. ¿De dónde obtiene principalmente los EPP para su trabajo cotidiano?

Se proveen en el lugar de trabajo $\square \quad$ Donaciones $\square \quad$ Llevan los propios trabajadores $\square \quad$ Otra $\square$

I4. ¿Existe en su lugar de trabajo algún protocolo de manejo para pacientes con alguno de los síntomas descriptos para COVID-I9?

Sí $\square \quad$ No $\square \quad$ No sabe $\square$

14.I. ¿Se cumple el mismo?

Sí $\square \quad$ No $\square \quad$ No sabe $\square$ 
I5. En caso de trabajar en institución con servicios de internación, ¿existe protocolo de aislamiento para nuevos pacientes que ingresan?

Sí $\square \quad$ No $\square \quad$ No sabe $\square$

I5. I. ¿Se cumple el protocolo?

Sí $\square \quad$ No $\square \quad$ No sabe $\square$

TRABAJO EN INSTITUCIONES PRIVADAS (En caso de no trabajar en instituciones privadas avance a la pregunta 24)

16. ¿Está realizando su trabajo en forma presencial?

Sí $\square \quad$ No $\square$

16. I. En el caso de estar desempeñando sus tareas laborales en forma virtual/online, ¿recibe los honorarios completos correspondientes?

Sí $\square \quad$ No $\square \quad$ No sabe/ no contesta $\square$

16.2. ¿Su empleador cumple con exceptuar de realizar tareas clínico/asistenciales a profesionales que se encuentren dentro de la población de riesgo para COVID-19 o a cargo de un menor? (Población de riesgo: embarazadas, comorbilidades)

Sí $\square \quad$ No $\square \quad$ No sabe / no contesta $\square$

16.3. En caso de estar exceptuado de asistir a su lugar de trabajo ¿su empleador continúa abonando su sueldo completo?

Sí $\square \quad$ No $\square \quad$ No sabe / no contesta $\square$

17. ¿Le resultan suficientes las medidas de desinfección tomadas en su ámbito laboral? (limpieza de consultorios con productos adecuados, limpieza de áreas de uso común entre profesionales, etc.)

Sí $\square \quad$ No $\square \quad$ No sabe / no contesta $\square$

18. Su ámbito de trabajo es:

Institución monovalente en Salud Mental $\square \quad$ Institución polivalente $\square \quad$ Otra $\square$

19. ¿En qué sector del ámbito se desempeña? (puede seleccionar más de una opción)

Guardia $\square \quad$ Servicio de internación $\square \quad$ Comunidad Terapéutica $\square \quad$ Consultorios Externos $\square \quad$ Hospital de día

Centro de salud $\square \quad$ Hogar de adultos mayores $\square \quad$ Otra $\square$

20. ¿Cuál de los siguientes elementos tanto del equipo de protección personal (EPP) como de higiene cuenta en su trabajo?

Barbijo $\square \quad$ Camisolín $\square \quad$ Guantes $\square \quad$ Protección ocular $\square$

Máscaras (protección plástica transparente que cubre la cara) $\square \quad$ Tapa nariz y boca

Acceso a agua potable y jabón entre pacientes $\square \quad$ Alcohol en gel $\square \quad$ Ninguno $\square \quad$ Otro $\square$

21. ¿De dónde obtiene principalmente los EPP para su trabajo cotidiano?

Se proveen en el lugar de trabajo $\square \quad$ Donaciones $\square \quad$ Llevan los propios trabajadores $\square \quad$ Otra $\square$

22. ¿Existe en su lugar de trabajo algún protocolo de manejo para pacientes con alguno de los síntomas descriptos para COVID-I9?

Sí $\square \quad$ No $\square \quad$ No sabe / no contesta $\square$

22. I. ¿Se cumple el mismo?

Sí $\square \quad$ No $\square \quad$ No sabe

23. En caso de trabajar en institución con servicios de internación, ¿existe protocolo de aislamiento para nuevos pacientes que ingresan?

Sí $\square \quad$ No $\square \quad$ No sabe $\square$

23.I. ¿Se cumple el protocolo?

Sí $\square \quad$ No $\square \quad$ No sabe $\square$

TANTO EN EL ÁMBITO PÚBLICOY/O PRIVADO: DESCRIPCIÓN (OPCIONAL)

24. ¿Ha sufrido algún acto de discriminación, por su condición de profesional de la salud, en la vía pública?

Sí $\square \quad$ No $\square \quad$ No sabe / no contesta $\square$

25. ¿Ha sufrido algún acto de discriminación en su vivienda?

Sí $\square \quad$ No $\square \quad$ No sabe / no contesta $\square$

26. ¿Ha sufrido alguna agresión verbal por su condición de profesional de la salud desde el inicio de la pandemia?

Sí $\square \quad$ No $\square \quad$ No sabe / no contesta $\square$

27. ¿Ha sufrido alguna agresión física por su condición de profesional de la salud desde el inicio de la pandemia?

Sí $\square \quad$ No $\square \quad$ No sabe / no contesta $\square$

28. Comentarios

Puede dejar comentarios y/o aportes sobre aspectos que no se hayan abordado en este cuestionario: 